\title{
A Novel Fusion between CDC42BPB and ALK Exon 20 in a Patient with Wild Type Gastrointestinal Stromal Tumor: a case report
}

\section{Wen Huang}

Zhongshan Hospital Fudan University https://orcid.org/0000-0003-1052-9955

\section{Wei Yuan}

Zhongshan Hospital Fudan University

\section{Lei Ren}

Zhongshan Hospital Fudan University

Chen Xu

Zhongshan Hospital Fudan University

\section{Rongkui Luo}

Zhongshan Hospital Fudan University

\section{Yuhong Zhou}

Zhongshan Hospital Fudan University

\section{Weiqi Lu}

Zhongshan Hospital Fudan University

Yingyong Hou ( $\sim 980455691 @ q q . c o m)$

Fudan University

\section{Case report}

Keywords: Gastrointestinal stromal tumors, ALK (D5F3) expression, ALK rearrangement, receptor tyrosine kinase

Posted Date: December 21st, 2020

DOl: https://doi.org/10.21203/rs.3.rs-131099/v1

License: (c) (i) This work is licensed under a Creative Commons Attribution 4.0 International License. Read Full License 


\section{Abstract \\ Background}

Gastrointestinal stromal tumors (GISTs) are the most common type of mesenchymal tumor in gastrointestinal tract $(\mathrm{Gl})$, with striking features of morphology and immunohistochemistry. Pathogenic activating mutations of the proto-Oncogene Kit are detected in $80-85 \%$ GISTs, while $5-7 \%$ of cases harbor activating mutations of platelet derived growth factor receptor alpha (PDGFRA). There are still some rare mutation types, including succinate dehydrogenase (SDH)-x, BRAF, RAS and NF1 mutations, or ETV6-NTRK3 fusion. Herein, we reported the first case of GIST with ALK gene rearrangement and ALK (D5F3) overexpression.

\section{Case presentation}

In this study, we described a 33-year-old female patient who appeared a massive space occupying lesion (with the maximum diameter of $22 \mathrm{~cm}$ ) in the stomach and was eventually diagnosed as Kit and PDGFRA wide type GIST. We unexpectedly found that GIST of this patient showed ALK (D5F3) overexpression and harbored a novel fusion CDC42BPB exon 24-ALK in exon 20.

\section{Conclusion}

This is the first case of GIST with both ALK rearrangement (CDC42BPB exon 24-ALK in exon 20) and ALK (D5F3) overexpression. This rare phenomenon might suggest that ALK (D5F3) immunohistochemistry could be a screening tool for ALK-rearranged GIST. In addition, ALK inhibitors could be a potential therapeutic target for patients (with ALK rearrangement), which should be confirmed further.

\section{Background}

Gastrointestinal stromal tumors (GISTs) are the most common mesenchymal tumors occurring within the GI tract which most frequently originate from the stomach [1]. The great majority of GISTs harbor mutations in the Kit or PDGFRA genes (the members of the type III tyrosine kinase receptors) [2]. The remaining $10 \%$ of GISTs do not harbor any mutation of the KIT and PDGFRA genes, and are designated as wild type GISTs [3].

The tyrosine kinase inhibitors (TKIs), especially imatinib, have been used as a standard first-line treatment for patients with localized and advanced GIST [4]. What's more, there are increasing literatures illustrating the differential responses to TKIs (or imatinib) in GIST patients with different mutations [5]. For example, the majority of GIST patients are imatinib-sensitive (especially with KIT exon 11 mutations), while GISTs harboring a mutation in KIT exon 17 or PDGFRA exon 18 (p.D842V) are confirmed as imatinib-resistant [6]. Additionally, TKIs are not effective for wild type GIST patients, especially the SDH- 
deficient cases [7]. Therefore, identifying gene mutations in different patients is critical to guide the therapy and improve the prognosis by matching targeted drugs to specific mutations [8]. It is also necessary to explore new therapeutic targets as alternative or first choice for patients with drug-resistant GIST.

GIST is a pleomorphic mesenchymal tumor which excretes CD117 (Kit protein) or CD34 as immunohistochemical agents [9]. Besides, DOG-1 is also considered to be a marker of GIST [10]. To our knowledge, most GISTs are ALK-negative, which is helpful to differentiate GISTs from other mesenchymal neoplasms, such as inflammatory myofibroblastic tumors (IMTs) (most of which are positive for ALK) [11]. ALK is a transmembrane receptor tyrosine kinase and its overexpression can be caused by gene fusion, mutations and amplification [12]. Previous researches demonstrated that ALK gene rearrangement, in which fusion of ALK and a second gene led to a new driver oncogene and served as a biomarker $[13,14]$. Until now, ALK rearrangement have been reported in various neoplasms, including pseudosarcomatous myofibroblastic proliferations [15], secretory carcinoma (SCs) [16] and non-small cell lung cancer (NSCLC) in China [17]. However, the relationship between the rearrangement of ALK and GIST has not been entirely clear. To date, only two studies have reported ALK overexpression status of GIST $[12,18]$; and only one literature has showed little on the clinical and molecular characteristics of GIST with ALK rearrangement [18]. Besides, no limited targeted RNA-seq results were reported in any case.

In this study, we presented a case of 33-year-old woman with wide type GIST, in which ALK (D5F3) was overexpressed. We also determined the prevalence of ALK gene rearrangement with fluorescence in situ hybridization (FISH), so as to identify specific fusion genes by next-generation sequencing (NGS) and targeted RNA sequencing, for a better analysis of a novel gene fusion in GIST.

\section{Case Presentation}

A 33-year-old female patient initially admitted to the hospital for routine antenatal testing and a massive space occupying lesions (with an estimated disaster of $20.5 \mathrm{~cm}$ ) located in the right upper abdomen was discovered incidentally on MRI. Subsequently this patient was transferred to another hospital for further examination. The computed tomography (CT) revealed a mass adherent to the stomach, which was also adjacent to the liver and pancreas (Fig. 1). The tumor and part of the stomach were surgically removed in July.2020. Through gross examination, the tumor was $22 \times 17 \times 14 \mathrm{~cm}$ in size and the cut surface of it showed yellowish white appearance (Fig. 2). Histologic examination revealed numerous spindle cells with coagulation necrosis and medium nuclear atypia, small nucleoli, and low mitotic activity (2 mitosis/ 50 HPF). No lymph node metastases were observed. Immunohistochemically, the spindle cells were positive for CD117, DOG-1, CD34, SDHA and SDHB (Fig. 3b-f), but negative for a-SMA, desmin and S100. Ki-67 was estimated to be $5 \%$. As a result, the diagnosis of NIH high-risk GIST was rendered. Molecular analysis revealed the absence of Kit (exon 9, 11, 13,17) and PDGFRA (exon 12,18), which also didn't harbor any mutations of K-ras (exon 2, 3, 4), B-raf (exon 15), N-ras (exon 2, 3, 4) and PIK3CA (exon 20), indicating a 
Kit and PDGFRA wide type GIST. Therefore, NGS was carried out to seek the possibility of new targets for therapeutic strategies.

Subsequently, genomic DNA was extracted from the unstained $4 \mu \mathrm{m}$ formalin fixed paraffin-embedded tissue and performed for NGS. The results of NGS analysis and structural variant analysis identified a novel rearrangement involving CDC42BPB exon 24 (chr14:103 417 527) and ALK exon 19 (chr10:29 446 465), which was suggested to encode an in-frame fusion protein containing the ALK kinase domain (Fig. 5a) [19]. As we know, exon 19 is a common breakpoint location for oncogenic ALK rearrangements $[16,17]$, but this is the first report of an ALK fusion with CDC42BPB.

In addition, RNA sequencing revealed that CDC42BPB-ALK fusion including ALK exons 20-29 with the predicted breakpoint in exon 19. The breakpoint of CDC42BPB involves exon 24, rendering fusion product of encompass exons 1-24 (Fig. 5b-c). The CDC42BPB-ALK fusion was in-frame. More importantly, this fusion product includes the intracytoplasmic C-terminal domain of ALK (exons 20-29) [20], containing the ALK kinase domain.

CDC42BPB-ALK fusion was screened out, and some types of ALK fusions were verified as a possible therapeutic target against ALK inhibitors in other cancers [21, 22], such as SC (with CTNNA1-ALK) and ALK fusion NSCLC. In order to further testify the ALK gene status, we performed FISH analysis using the Vysis ALK Break Apart FISH Probe kit (Abbott Molecular, Des Plaines, IL, USA). And split signals for ALK could be detected in $90 \%$ tumor cells, indicating the ALK gene breaking (Fig. 4). Additionally, ALK protein expression was confirmed by immunohistochemistry (two different antibody clones, D5F3 and 5A4 were used). However, ALK was strongly stained only by the antibody of D5F3 (Fig. 3g). Generally speaking, these results mentioned above suggested that this case was a GIST (negative for other known GIST driver genes, such as Kit, PDGFRA, BRAF, SDHx and others) with ALK gene rearrangement, which was likely to function as the oncogenic driver in this tumor.

Although wild type GIST has been found to be insensitive to TKIs, especially in SDH-deficient GIST, there are still a few patients who can benefit from the TKIs treatment. Immunohistochemical shows SDHB positive in this patient, so postoperative adjuvant imatinib therapy can be tried. However, this patient is still in lactation and declines medical treatment. Therefore, close follow-up was suggested for this patient. Now 4 months after resection (from July.2020), this patient is alive and well, with no sign of recurrence.

\section{Discussion And Conclusions}

Here, we described a case with a huge tumor located in the stomach. Morphological feature revealed that tumor tissue from the spindle cells and collagen fiber bundles cutting through. Besides, IHC results showed that the spindle cells expressed CD117, DOG1, CD34, SDHA, SDHB, while a-SMA and other markers were negative. Given the immunophenotype of Cajal cell differentiation, which was confirmed to express CD117 and DOG-1 [23], as well as the location of this lesion, the diagnosis of GIST was first taken into consideration. 
In order to further confirm the diagnosis of GIST, genetic analysis was carried out, and the results revealed a KIT and PDGFRA wild type GIST. NGS sequencing of a recent study claimed that [24] TP53, RB and ALK mutations could be detected in 72 Chinese patients with quadruple-negative GISTs (devoid of KIT, PDGFRA, BRAF, and SDH alterations). Besides, as it is known to all, wild type GIST is not sensitive to TKIs. Previous studies have suggested that the resistance to imatinib can be restored by other gene inhibitions, including FGFR3 [25] and NTRK3 [26]. Therefore, NGS was performed to identify driver mutations in GIST so as to find the potential therapeutic targets. To our surprise, a novel CDC42BPB-ALK fusion type (CDC42BPB exon 24-ALK in exon 20) with involvement of ALK exon 20 was identified. The function of the fusion protein is still unknown for the reason that CDC42BPB has not been reported as a fusion partner of ALK in any ALK-rearranged tumor. The result of RNA sequencing showed that CDC42BPB gene might be a fusion partner to ALK in some GIST cases. CDC42BPB, a member of the small Rho GTPase family [27], was located on 14q32.3 chromosome by FISH [28]. Shivashankar et al [29] discovered that CDC42BPB was a kind of non-cancer associated gene, and would serve as potential drug target and diagnostic or prognostic markers if the cancer association was established. In addition, there is no previous report of any causal mutation of CDC42BPB in cancer. However, ALK fusion after exon 20 on the ALK side, which includes the complete ALK kinase domain, has been reported to activate a carcinogenic kinase in various ALK-rearranged tumors [30], including NSCLC [19], IMT [31], peritoneal mesothelioma and various other carcinomas [32]. Previous study demonstrated that wild type GIST with the ETV6-NTRK3 fusion gene could be suppressed by ALK inhibitors, which could also inhibit NTRK3 [33]. Similarly, we supposed that ALK inhibitors might serve as a new strategy for the targeting therapy of GIST in this case, which required further exploration and research.

FISH analysis (using FISH break-apart probes) and IHC methods were performed to identify ALK gene status. We found that a significant proportion of ALK genes were split. According to the relevant researches, the relation between the expression of ALK protein and ALK rearrangement was not sure. In 2020, Jun et al [12] reported a case of GIST with PDGFRA p. D842V mutation, which showed ALK protein overexpression (both of D5F3 and 5A4 clones) but lacked ALK rearrangement by FISH analysis. However, Lei et al [18] presented GIST with an ALK gene rearrangement (PPP1R21-ALK) which was examined by NGS. One possibility accounts for this confusing phenomenon is that some cases harbor cryptic structural variants that cannot be resolved by FISH [34]. Besides, according to the standard methods which were advocated by the US Food and Drug Administration (FDA), as well as the China Food and Drug Administration (CFDA), FISH basing on break-apart FISH probes and IHC using D5F3 antibody were standard methods for ALK arrangement detection in NSCLC [30]. In our study, we found that ALK overexpression could only be observed with the usage of D5F3 antibody, and this ALK (D5F3)-positive GIST had ALK gene rearrangement. Our current findings indicated that the use of different immunohistochemical antibody clones of ALK could also affect results, and the in-frame rearrangement might result in expression of a CDC42BPB-ALK fusion protein.

Our findings suggest that ALK fusion in the GIST starts at the canonical exon 20, and this type of ALK fusion has been reported as a possible therapeutic target in other cancers $[35,36]$. Therefore, we supposed that ALK inhibitors would be a therapeutic option for patients with ALK-rearranged GIST who 
were insensitive to or resistant to TKIs. In this case, given that Kit and PDGFRA wild type GIST was proved to be not sensitive to TKIs, and this female patient is still in lactation, follow-up was suggested for this patient. During 4 months of following up, there was no recurrence after excision. If this patient develops recurrence/metastases in the future, it would seem reasonable to try targeted therapy using ALK inhibitors. However, the frequency of ALK rearrangement in GIST appears to be low and there were too few samples of patient cohorts. To date, ALK fusion has not been examined in GIST with intention (even using ALK immunohistochemistry for a preliminary screening). Thus, further research needs to be done in larger patient cohorts.

In conclusion, we identified a GIST with a novel fusion of CDC42BPB-ALK. Our results, combining with previously described 2 cases of GIST (with ALK gene rearrangement or ALK overexpression), also expanded the molecular spectrum of this tumor beyond the well-known GIST driver genes. These oncogenic events may have implications for therapeutic targets in patients with ALK rearranged GIST, which deserves our further investigation.

\section{Abbreviations}

GIST

Gastrointestinal stromal tumor;

GI

Gastrointestinal tract;

PDGFRA

Platelet derived growth factor receptor alpha;

$\mathrm{SDH}$

Succinate dehydrogenase;

TKIs

Tyrosine kinase inhibitors;

IMTs

Inflammatory myofibroblastic tumors;

SC

Secretory carcinoma;

NSCLC

Non-small cell lung cancer;

IHC

Immunohistochemistry;

$\mathrm{FISH}$

Fluorescence in situ hybridization;

NGS

Next-generation sequencing;

CT

Computed tomography; 
PCR

Polymerase chain reaction;

\section{Declarations}

\section{Acknowledgements}

The Authors sincerely express their thanks for the financial support of the funds for our research (see under Funding) and grate fully acknowledge our colleagues and many individuals for their help during the study processes.

\section{Authors' contributions}

WH and WY conducted the studies, and are the primary Authors of this article. WH and WY performed the related experiments, and WH drafted the article. RL, CX and Rk L collected specimens and the history data. Yh Z developed treatment plans for the patients and follow-up. Wq $\mathrm{L}$ summarized the clinical and histologic data. Yy $\mathrm{H}$ conceived the project, revised the article, obtained the funding, and designed the research. All Authors met the authorship criteria and read and approved the final article.

\section{Funding}

This work was financially supported by Shanghai 305 Municipal Commission of Science and Technology (No. 19441904000), Shanghai Municipal Key 306 Clinical Specialty (No. shslczdzk01302).

\section{Availability of data and materials}

All data generated or analyzed during this study are included in this published article.

\section{Ethics approval and consent to participate}

This study was approved by the Institutional Review Board (IRB) of Zhongshan Hospital Fudan University. Informed consent was obtained from the patient.

\section{Consent for publication}

Written informed consent was obtained from the patients for the publication of this case report and any accompanying images.

\section{Competing interests}

The authors declare that they have no competing interests.

\section{References}


1. Mayr P, Märkl B, Agaimy A, Kriening B, Dintner S, Schenkirsch G, et al. Malignancies associated with GIST: a retrospective study with molecular analysis of KIT and PDGFRA. Langenbecks Arch Surg. 2019;404(5):605-13.

2. Vankova B, Behenska K, Bauer M, Sedivcova M, Daumova M, Agaimy A, et al. Morphological features useful in the differential diagnosis between undifferentiated carcinoma and ga strointestinal stromal tumor. Ann Diagn Pathol. 2020;46:151527.

3. Huss S, Künstlinger H, Wardelmann E, Kleine MA, Binot E, Merkelbach-Bruse S, et al. A subset of gastrointestinal stromal tumors previously regarded as wild-type tumors carries somatic a ctivating mutations in KIT exon 8 (p.D419del). Mod Pathol. 2013;26(7):1004-12.

4. Casali PG, Abecassis N, Bauer S, Biagini R, Bielack S, Bonvalot S, et al. Gastrointestinal stromal tumours: ESMO-EURACAN Clinical Practice Guidelines for diagnosis, treatment and follow-up. Ann Oncol. 2018;29 Suppl 4:iv68-iv78.

5. Corless CL, Schroeder A, Griffith D, Town A, McGreevey L, Harrell P, et al. PDGFRA mutations in gastrointestinal stromal tumors: frequency, spectrum and in vitro sensitivity to imatinib. J Clin Oncol. 2005;23(23):5357-64.

6. Cassier PA, Fumagalli E, Rutkowski P, Schöffski P, Van Glabbeke M, Debiec-Rychter M, et al. Outcome of patients with platelet-derived growth factor receptor alpha-mutated gastrointestinal stromal tumors in the tyrosine kinase inhibitor era. Clin Cancer Res. 2012;18(16):4458-64.

7. Boikos SA, Stratakis CA. The genetic landscape of gastrointestinal stromal tumor lacking KIT and PDGFRA mutations. Endocrine. 2014;47(2):401-8.

8. Park J, Yoo HM, Sul HJ, Shin S, Lee SW, Kim JG. Genetic Characterization of Molecular Targets in Korean Patients with Gastrointestinal Stromal Tumors. J Gastric Cancer. 2020;20(1):29-40.

9. Şentürk M, Yıldırım MA, Çakır M, Kişi Ö. Clinicopathologic and Surgical Characteristics Study of 151 Cases of GIST. J Gastrointest Cancer. 2020.

10. West RB, Corless CL, Chen X, Rubin BP, Subramanian S, Montgomery K, et al. The novel marker, DOG1, is expressed ubiquitously in gastrointestinal stromal tumors irrespective of KIT or PDGFRA mutation status. Am J Pathol. 2004;165(1):107-13.

11. Kataoka TR, Yamashita N, Furuhata A, Hirata M, Ishida T, Nakamura I, et al. An inflammatory myofibroblastic tumor exhibiting immunoreactivity to KIT: a case report focusing on a diagnostic pitfall. World J Surg Oncol. 2014;12:186.

12. Fan J, Yang M, Huang B, Wang Z, Luo D, Zhang J, et al. ALK expressed in a gastrointestinal stromal tumor harboring PDGFRA p. D842V mutation:a case report. Diagn Pathol. 2020;15(1):8.

13. Soda M, Choi YL, Enomoto M, Takada S, Yamashita Y, Ishikawa S, et al. Identification of the transforming EML4-ALK fusion gene in non-small-cell lung cancer. Nature. 2007;448(7153):561-6.

14. Perner S, Wagner PL, Demichelis F, Mehra R, Lafargue CJ, Moss BJ, et al. EML4-ALK fusion lung cancer: a rare acquired event. Neoplasia. 2008;10(3):298-302.

15. Albores-Saavedra J, Manivel JC, Essenfeld H, Dehner LP, Drut R, Gould E, et al. Pseudosarcomatous myofibroblastic proliferations in the urinary bladder of children. Cancer. 1990;66(6):1234-41. 
16. Sasaki E, Masago K, Fujita S, Suzuki H, Hanai N, Hosoda W. Salivary Secretory Carcinoma Harboring a Novel ALK Fusion: Expanding the Molecular Characterization of Carcinomas Beyond the ETV6 Gene. Am J Surg Pathol. 2020;44(7):962-69.

17. Fei X, Zhu L, Zhou H, Qi C, Wang C. A Novel Intergenic Region between CENPA and DPYSL5-ALK Exon 20 Fusion Variant Responding to Crizotini b Treatment in a?Patient with Lung Adenocarcinoma. J Thorac Oncol. 2019;14(9):e191-e93.

18. Zhao L, Nathenson MJ, Nowak JA, Fairweather M, Hornick JL. ALK rearrangement in a gastrointestinal stromal tumor of the small bowel. Histopathology. 2020.

19. Shimizu N, Akashi Y, Fujii T, Shiono H, Yane K, Kitahara T, et al. Use of ALK Immunohistochemistry for Optimal Therapeutic Strategy of Pulmonary Large-cell Neuroendocri ne Carcinoma and Identification of a Novel KIF5B-ALK Fusion Oncokinase. Anticancer Res. 2019;39(1):413-20.

20. Le AT, Varella-Garcia M, Doebele RC. Oncogenic fusions involving exon 19 of ALK. J Thorac Oncol. 2012;7(12):e44.

21. Yakirevich E, Resnick MB, Mangray S, Wheeler M, Jackson CL, Lombardo KA, et al. Oncogenic ALK Fusion in Rare and Aggressive Subtype of Colorectal Adenocarcinoma as a Potential Thera peutic Target. Clin Cancer Res. 2016;22(15):3831-40.

22. Mano H. ALKoma: a cancer subtype with a shared target. Cancer Discov. 2012;2(6):495-502.

23. Dirnhofer S, Zimpfer A, Went P. [The diagnostic and predictive role of kit (CD117)]. Ther Umsch. 2006;63(4):273-8.

24. Wang S, Sun RZ, Han Q, Wang SY, Wang EH, Liu Y. Genomic Study of Chinese Quadruple-negative GISTs Using Next-generation Sequencing Technology. Appl Immunohistochem Mol Morphol. 2020.

25. Javidi-Sharifi N, Traer E, Martinez J, Gupta A, Taguchi T, Dunlap J, et al. Crosstalk between KIT and FGFR3 Promotes Gastrointestinal Stromal Tumor Cell Growth and Drug Resistan ce. Cancer Res. 2015;75(5):880-91.

26. Brenca M, Rossi S, Polano M, Gasparotto D, Zanatta L, Racanelli D, et al. Transcriptome sequencing identifies ETV6-NTRK3 as a gene fusion involved in GIST. J Pathol. 2016;238(4):543-9.

27. Pichaud F, Walther RF, Nunes de Almeida F. Regulation of Cdc42 and its effectors in epithelial morphogenesis. J Cell Sci. 2019;132(10).

28. Moncrieff CL, Bailey ME, Morrison N, Johnson KJ. Cloning and chromosomal localization of human Cdc42-binding protein kinase beta. Genomics. 1999;57(2):297-300.

29. Nagaraj SH, Reverter A. A Boolean-based systems biology approach to predict novel genes associated with cancer: Application t o colorectal cancer. BMC Syst Biol. 2011;5:35.

30. Liu Y, Wu S, Shi X, Liang Z, Zeng X. ALK detection in lung cancer: identification of atypical and cryptic ALK rearrangements using an optimal algorithm. Journal of Cancer Research and Clinical Oncology. 2020;146(5):1307-20.

31. Lawrence B, Perez-Atayde A, Hibbard MK, Rubin BP, Dal Cin P, Pinkus JL, et al. TPM3-ALK and TPM4ALK oncogenes in inflammatory myofibroblastic tumors. Am J Pathol. 2000;157(2):377-84. 
32. Huang H. Anaplastic Lymphoma Kinase (ALK) Receptor Tyrosine Kinase: A Catalytic Receptor with Many Faces. Int J Mol Sci. 2018;19(11).

33. Huss S, Elges S, Trautmann M, Sperveslage J, Hartmann W, Wardelmann E. Classification of KIT/PDGFRA wild-type gastrointestinal stromal tumors: implications for therapy. Expert Rev Anticancer Ther. 2015;15(6):623-8.

34. Acosta AM, Demicco EG, Dal Cin P, Hirsch MS, Fletcher CDM, Jo VY. Pseudosarcomatous myofibroblastic proliferations of the urinary bladder are neoplasms characterized b y recurrent FN1ALK fusions. Mod Pathol. 2020.

35. Yoshida T, Oya Y, Tanaka K, Shimizu J, Horio Y, Kuroda H, et al. Differential Crizotinib Response Duration Among ALK Fusion Variants in ALK-Positive Non-Small-Cell Lu ng Cancer. J Clin Oncol. 2016;34(28):3383-9.

36. Lin JJ, Shaw AT. Differential Sensitivity to Crizotinib: Does EML4-ALK Fusion Variant Matter? J Clin Oncol. 2016;34(28):3363-5.

\section{Figures}

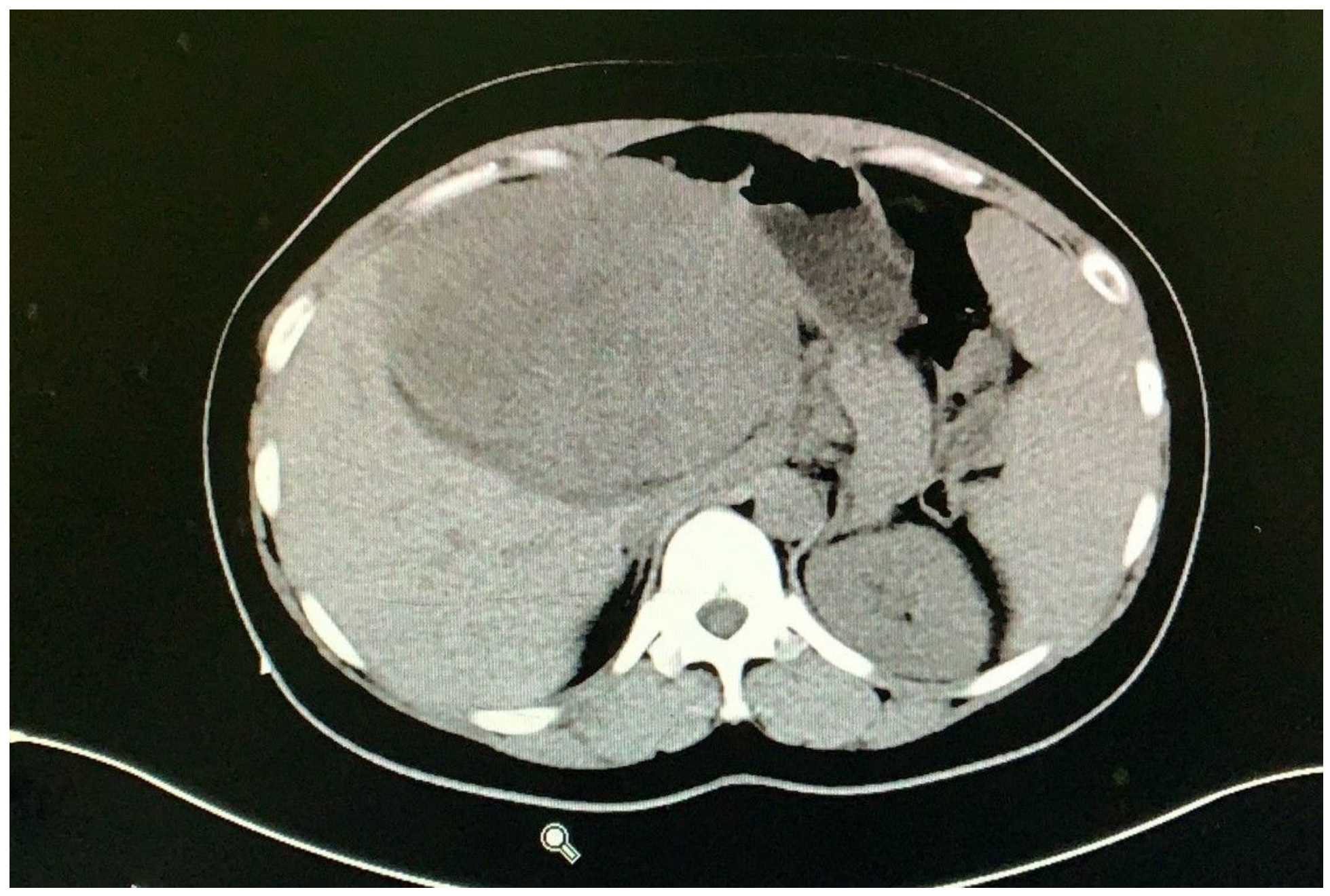

Figure 1 
Abdominal CT scan shows a massive space occupying lesion, which was related to gastric wall.

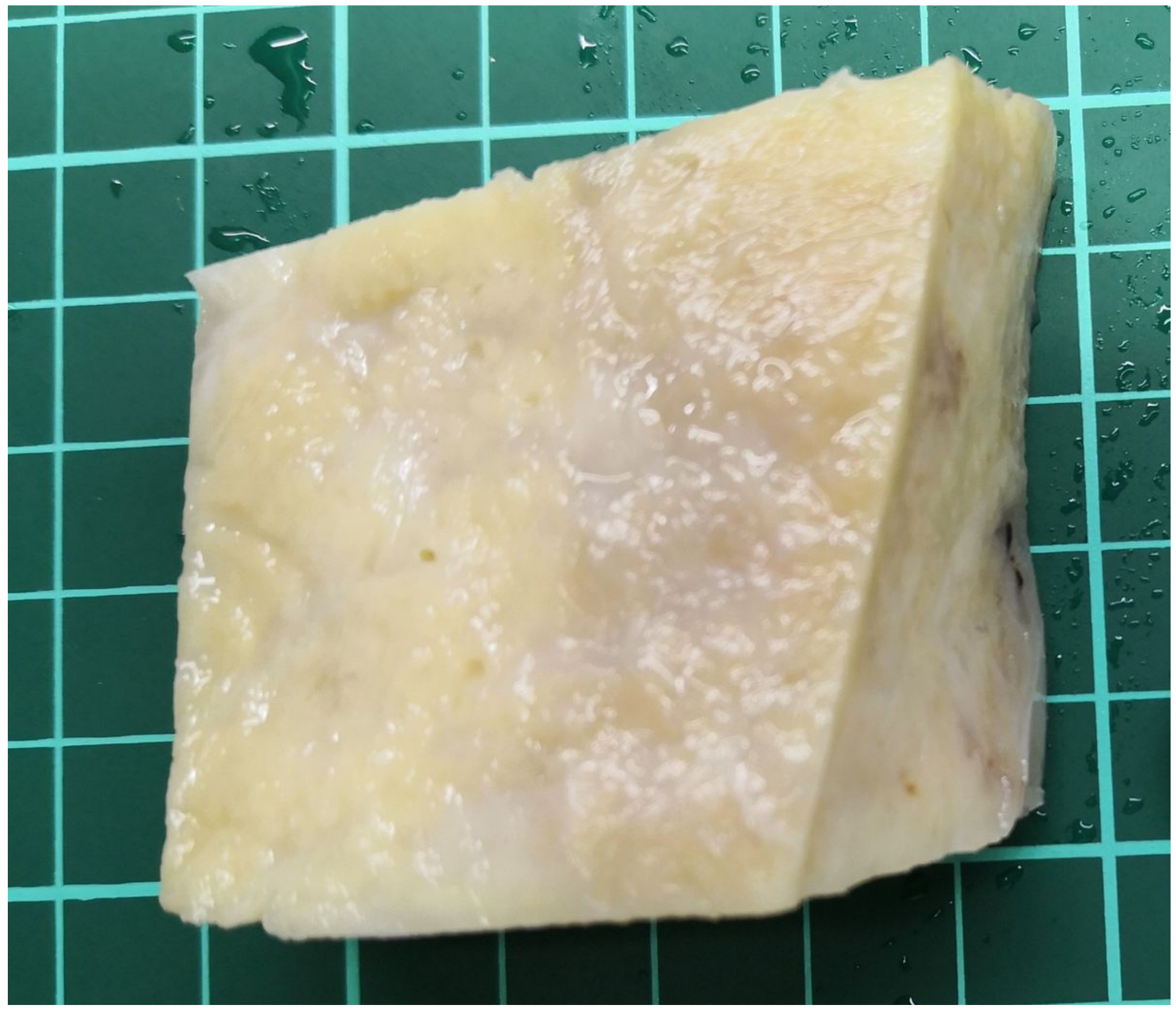

Figure 2

Gross image The cut surface of this tumor has yellowish white appearance. 

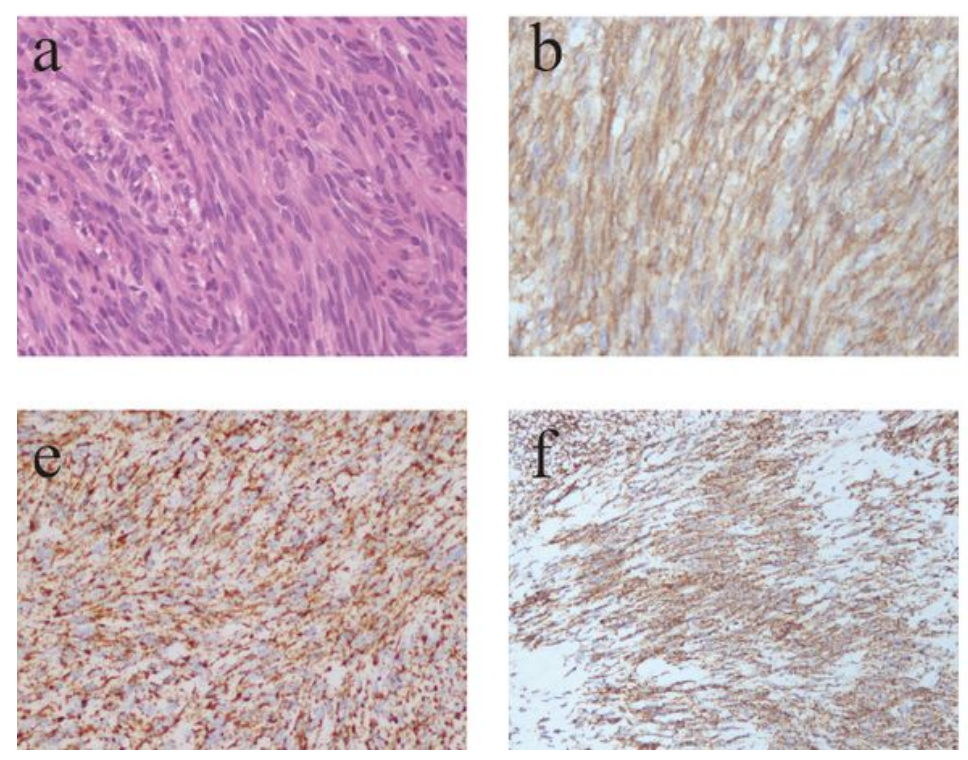
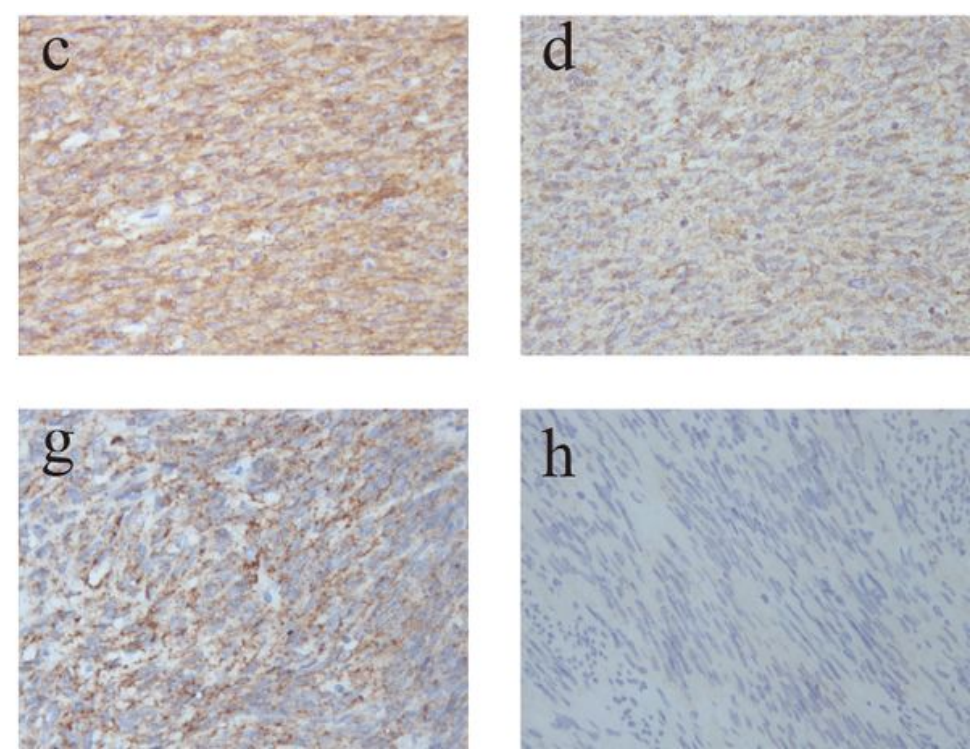

Figure 3

Section stained with hematoxylin and eosin (H\&E) (a, 400x). Immunohistochemistry staining (b h) indicated that tumor cells were positive for CD117 (b, 400x), Dog1 (c, 400x), CD34 (d, 400x), SDHA (e, 400x), SDHB (f, 400x) and ALK D5F3 (g, 400x), but negative for ALK 5A4 (h, 400x).

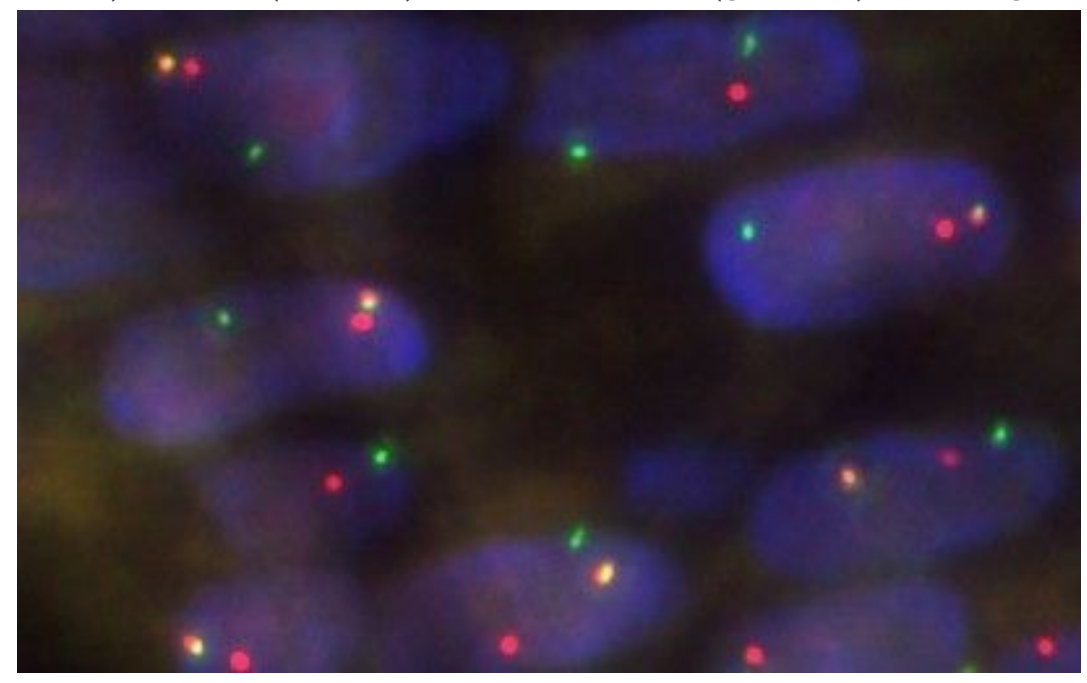

\section{Figure 4}

Fluorescence in situ hybridization (FISH) analysis using the FISH break-apart probes shows split signals for ALK (red, 3'; green, 5'). 

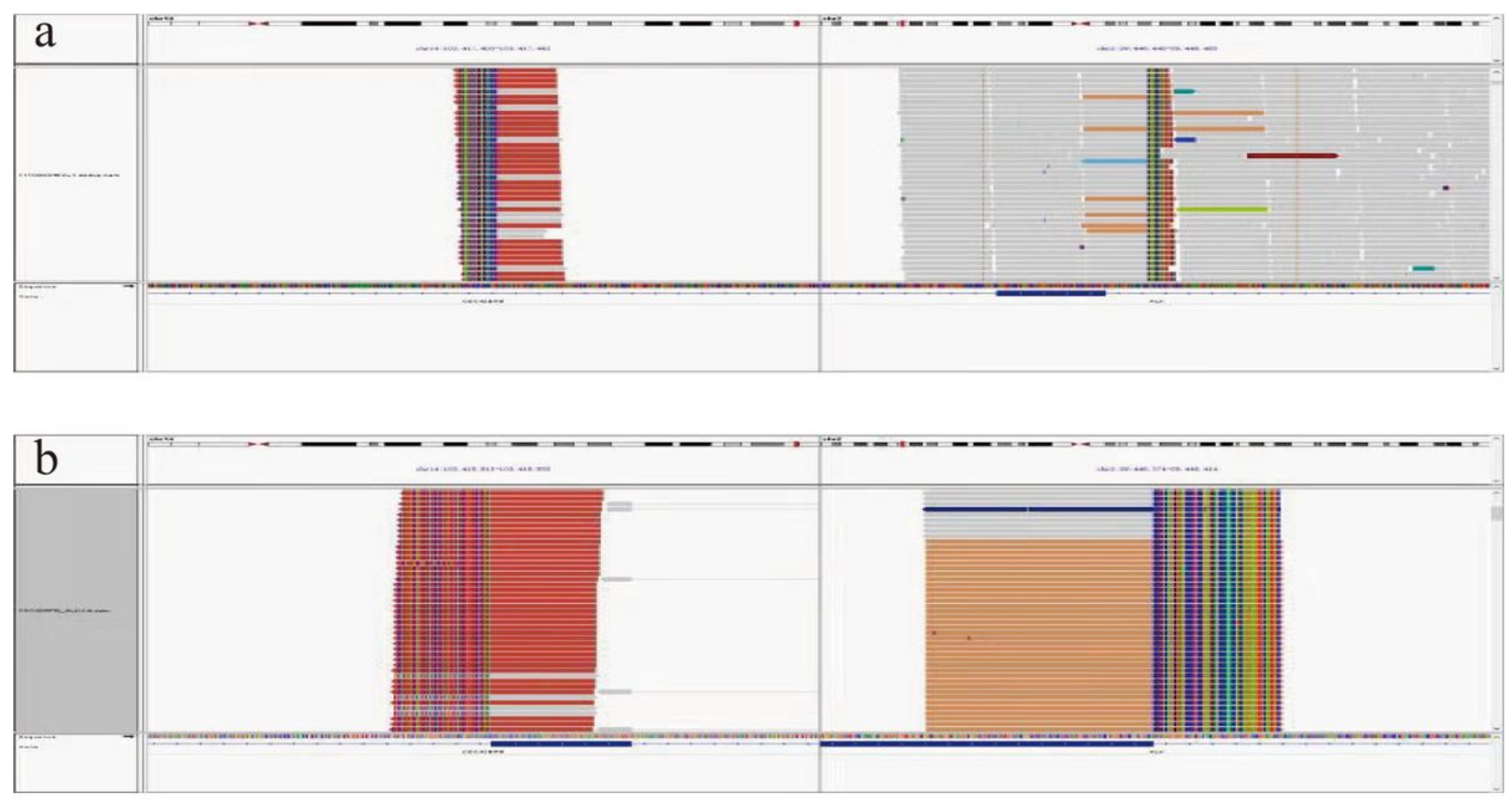

C

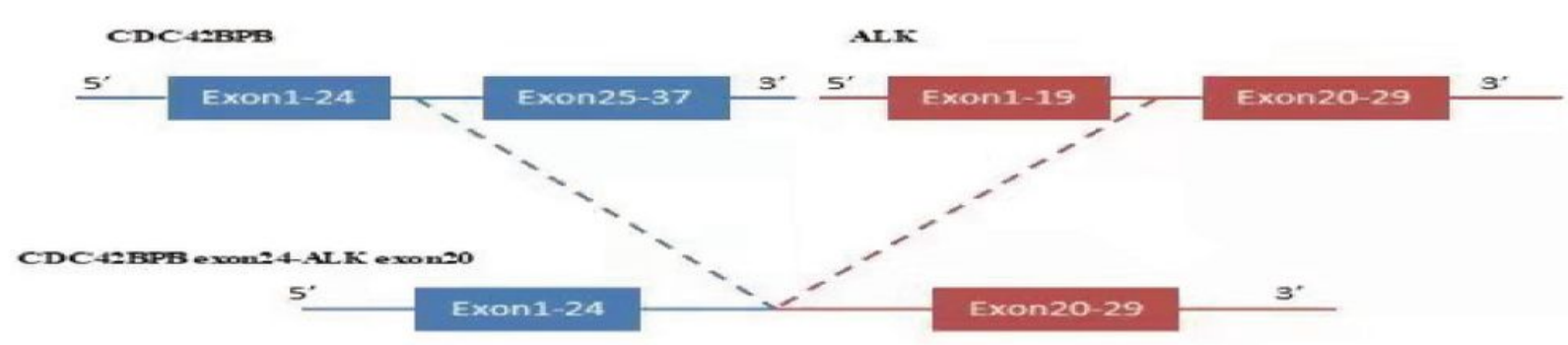

Figure 5

a A novel rare ALK rearrangement of CDC42BPB-ALK (exon 24: exon 20) was identified by Targeted nextgeneration sequencing (NGS). b A novel ALK fusion of CDC42BPB-ALK (exon 24: exon 20) was identified by RNA sequencing. $c$ A novel intergenic region between $C D C 42 B P B$ and receptor tyrosine kinase gene (ALK) exon 20 fusion variant was identified. 